\title{
Adaptive Sliding Mode Control Based On Offshore Doubly Fed Induction Generator for Wind Turbines
}

\author{
Feng Yang \\ School of Automation \\ UESTC \\ Chengdu, China \\ fyang@uestc.edu.cn
}

\author{
Shengshan Li \\ School of Automation \\ UESTC \\ Chengdu, China \\ liss1990liss@sina.com
}

\author{
Lei Wang \\ School of Energy Science and \\ Engineering \\ UESTC \\ Chengdu, China
}

\author{
YongDuan Song \\ School of Energy Science \\ and Engineering \\ UESTC \\ Chengdu, China
}

\author{
Qingwang Song \\ School of Automation \\ UESTC \\ Chengdu, China
}

\author{
Yuxing Wang \\ Mengcun County Science \\ and Technology Bureau \\ Cangzhou, China
}

\author{
Xiaoqiang Du \\ School of Energy Science \\ and Engineering \\ UESTC \\ Chengdu, China
}

\begin{abstract}
This paper deals with the speed control of a variable speed double-fed induction generator (DFIG)-based wind turbine. These variable speed control systems have several advantages over the traditional wind turbine, such as the increase in the energy capture and the reduction of the mechanical stress. To achieve the maximum power point tracking (MPPT) and improve the efficiency of a wind turbine, an adaptive sliding mode control via backstepping is proposed. Traditionally, wind turbine systems using mainly proportional integral (PI) controllers. However, such kinds of controller do not adequately handle some inaccuracies mainly leading to non-optimal power extraction. These may decrease wind turbine performances. Therefore, using robust control, such as adaptive sliding mode control via backstepping, will allow one to directly track the DFIG torque leading to maximum power extraction. Finally, simulation results are demonstrated to validate the proposed controllers.
\end{abstract}

Keywords-DFIG; adaptive sliding mode control; MPPT

\section{INTRODUCTION}

Due to reduction of fossil fuels and environmental pollutions, wind is recognized worldwide as a cost-effective, environment-friendly solution to energy shortage [1]. Wind energy conversion is the fastest-growing energy source among the new power generation sources in the world and this tendency should remain for some time. During 2011, an estimated 40GW of wind power capacity was put into operation, more than any other renewable technology, increasing global wind capacity by $20 \%$ to approximately 238GW .Over the period from 2006 to 2011, annual growth rates of cumulative wind power capacity averaged $26 \%$ [2].

Solution to various wind energy conversion problems, as yielded by wind energy system (WES) exploitation experience and grid integration, require the wide use of different control methods [3]. In this paper, a DFIG unit is embedded into the system to enhance the generated power quality. This is achieved by regulating the DFIG speed properly to maintain constant speed of the generator over wide range of wind speed.

This work was supported in part by the National High Technology Research and Development Program ("863") of China (SS2012AA052302) and in part by National Natural Science Foundation of China(No. 609704052 No. 61134001 and No. 51205046).
Note that DFIG has been widely used in high performance servo drives where a fast and accurate torque response is required because of their inherent advantages such as low inertia, high efficiency and high power density compared to other turbines with the same capacity. The main task for control system is to regulate the DFIG speed to enhance the power quality.

Reference [4, 5]-a second-order sliding mode control, PI-sliding mode controller is discussed. This method is simple but doesn't have a satisfactory robustness because of nonlinearity of the system, specially, with respect to stochastic inherent of wind that increases uncertainties. Adaptive sliding mode control via backstepping approach robustness to uncertainties is improved but it has the drawback of chattering effect, which might be harmful for the system. A trade-off exists between energy captured and high-frequency loading on the rotor to find an optimal operating condition.

The rest of the paper is organized as follow. Section II is dedicated to modeling framework of the considered turbine model and generator model. This model is used for the adaptive sliding mode control law design and stability analysis, as detailed in Section III. Section IV contains simulation analysis. Conclusion are grouped together in Section V.

\section{SYSTEM MODELING}

\section{A. Wind Turbine Aerodynamic Model}

The wind turbine dynamics are given by the popular two-mass model [6] as

$$
\left\{\begin{array}{l}
J_{w} \dot{\omega}_{w}=T_{a}-K_{w} \omega_{w}-B_{w} \theta_{w}-T_{l s} \\
J_{g} \dot{\omega}_{g}=T_{h s}-K_{g} \omega_{g}-B_{g} \theta_{g}-T_{e m}
\end{array}\right.
$$

where $J_{w}$ is the rotor inertia, $T_{l s}$ is the low-speed shaft torque, $K_{r}$ is the rotor external damping, $J_{g}$ is the generator inertia, 
$T_{h s}$ is the high-speed shaft torque, $K_{g}$ is the generator external damping, $B_{g}$ is the generator external stiffness, $B_{w}$ is the rotor external stiffness and $K_{l s}$ is the low-speed damping .

The ideal gearbox ratio is defined as

$$
n_{g}=T_{l s} / T_{h s}=\omega_{g} / \omega_{w}
$$

A single-mass for the wind turbine can be define as

$$
\begin{gathered}
J_{T} \dot{\omega}_{w}=T_{a}-K_{T} w_{w}-B_{T} \theta_{w}-T_{g} \\
\left\{\begin{array}{l}
J_{T}=J_{w}+n_{g}^{2} J_{g} \\
K_{T}=K_{W}+n_{g}^{2} K_{g} \\
B_{T}=B_{w}+n_{g}^{2} B_{g} \\
T_{g}=n_{g} T_{e m}
\end{array}\right.
\end{gathered}
$$

where

\section{B. Generator Model}

DFIG-based wind turbines, will offer several advantages including variable speed operation, as four-quadrant active and reactive power capabilities. The main merit of the DFIG is its capability to deliver constant voltage and frequency output for $\pm 30 \%$ speed variation around conventional synchronous speed. Then, such system result in lower converter cost and lower power losses compared to a system based on a fully fed synchronous generator with full-rated converter. A schematic diagram of a DFIG-based generation system is Fig. 3. This figure describes the DFIG-based wind turbine system main components: the mechanical part (turbine, gear, pitch drive) and the DFIG which may exchange power with the grid not only through the stator but also through the rotor [7].

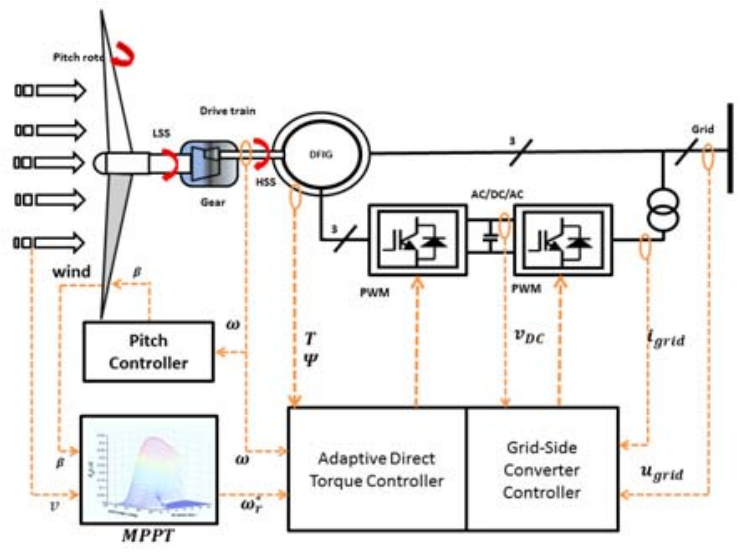

Figure 1. Proposed control structure

The generator use Current-mode control. The rotor current is split into two orthogonal components $\alpha$ and $\beta$. The control system is usually defined in the synchronous $\alpha \beta$ frame fix to the stator flux. For the proposed control strategy, the generator dynamic model written in a synchronously rotating frame $\alpha \beta$ is given by [8].

$$
\left\{\begin{array}{l}
d i_{r \alpha} / d t=a \psi_{s \alpha}+b \omega_{g} \psi_{s \beta}-c i_{r \alpha}+h u_{r \alpha} \\
d i_{r \beta} / d t=a \psi_{s \beta}-b \omega_{g} \psi_{s \alpha}-c i_{r \beta}+h u_{r \beta} \\
d \psi_{s \alpha} / d t=-e \psi_{s \alpha}-n_{p} \omega_{g} \psi_{s \beta}+f i_{r \alpha} \\
d \psi_{s \beta} / d t=-e \psi_{s \beta}+n_{p} \omega_{g} \psi_{s \alpha}+f i_{r \beta}
\end{array}\right.
$$

where $a=L_{m} R_{s} / \sigma L_{r} L_{s}^{2} ; \quad h=1 / \sigma L_{r} ; \quad b=n_{p} L_{m} / \sigma L_{s} L_{r}$; $c=L_{m}^{2} R_{s}+L_{s}^{2} R_{r} / \sigma L_{r} L_{s}^{2} ; e=R_{s} / L_{s} ; f=R_{s} L_{m} / L_{s}$.

The electromagnetic torque $T_{e m}$ is given by

$$
T_{e m}=3 n_{p} L_{m} / 2 J_{g} L_{s d}\left(\psi_{s \beta} i_{r \alpha}-\psi_{s \alpha} i_{r \beta}\right)
$$

\section{CONTROLler DESIGn AND StABILITy ANAlysis}

\section{A. Control strategy}

The generator torque is computed as a tabulated function of the filtered generator speed, incorporating four control region: 1, 2, 2.5, and 3 [9]. Region 1 is a control region before cut-in wind speed, where the generator torque is zero. In region 2, where input wind speed is below rated speed, pitch is held constant and generator torque is controlled by commanding a demand torque to maximize energy capture. Here, the generator torque is proportional to the square of the filtered generator speed to maintain a optimal tip-speed ratio. In region 3 , where wind speed is above rated speed, generator torque is held constant and blade-pitch control maintains rated power and rotor speed by shedding excess aerodynamic power. Region 2.5 is a linear transition between Regions 2 and 3 with a torque slope corresponding to the slope of an induction machine.

In regions 2-3, the demand torque is give by

$$
T(\omega)=\left\{\begin{array}{lr}
k \omega^{2} & \text { Region2 } \\
T_{1}+\frac{T_{\text {rated }}-T_{1}}{w_{\text {rated }}-w_{1}} & \text { Region2.5 } \\
T_{\text {rated }} & \text { Region3 }
\end{array}\right.
$$

\section{B. Controller design and stability analysis}

In order to facilitate the calculation, then from (1) (2) (4) (6) we have

$$
J_{T} \dot{\omega}_{w}=K_{o p t} w_{w}^{2}-K_{T} w_{w}-B_{T} \theta_{w}-n_{g} k \delta
$$

where $K_{\text {opt }}=\rho \pi R^{5} C_{p \max } / 2 \lambda_{\text {opt }}^{3}, \delta=\left(\psi_{s \beta} i_{r \alpha}-\psi_{s \alpha} i_{r \beta}\right)$

In order to achieve the mechanical part and the electric part of the generator isolation, is given as follows new state variable

$$
\left\{\begin{array}{l}
T=\psi_{s \alpha} i_{r \beta}-\psi_{s \beta} i_{r \alpha} \\
\psi=\left(\psi_{s \alpha}^{2}+\psi_{s \beta}^{2}\right) / 2 \\
x=\psi_{s \alpha} i_{r \alpha}+\psi_{s \beta} i_{r \beta}
\end{array}\right.
$$

where $T$ is the virtural torque, $T$ is relationship to the actual torque $T_{e}=k_{g} T ; \psi$ is virtual flux; $\psi$ is relationship to 
the actual torque $\psi=1 / 2 \psi_{s}^{2}$.

The DFIG model can be divided into mechanical parts and electrical parts. Assuming a perfectly rigid low-speed shaft, $B_{T}$ is very low, it can be neglected.

From (5),(6),(8)and(9), we have

$$
\left\{\begin{array}{l}
d \omega_{w} / d t=K_{o p t} \omega_{w}^{2} / J_{T}-K_{T} \omega_{w} / J_{T}-k n_{g} T / J_{T} \\
d T / d t=-2 b n_{g} \omega_{w} \psi-(e+c) T-n_{p} n_{g} \omega_{w} X+h u_{T}
\end{array}\right.
$$

where $u_{T}=\psi_{s \alpha} u_{r \beta}-\psi_{s \beta} u_{r \alpha}$.

$$
\left\{\begin{array}{l}
d \psi / d t=-2 e \psi+f x \\
d x / d t=2 a \psi-(e+c) x+n_{p} n_{g} \omega_{w} T+f I+h u_{\psi}+D
\end{array}\right.
$$

where $u_{\psi}=\psi_{s \alpha} u_{r \alpha}+\psi_{s \beta} u_{r \beta} ; \quad I=i_{s \alpha}^{2}+i_{s \beta}^{2}, \quad D \quad$ is the disturbance.

For the mechanical parts, $T$ is the virtual torque, $u_{T}$ is assumed to be a virtual torque voltage, for the electrical parts, $u_{\psi}$ is the virtual flux voltage.

Now, adaptive variable structure backstepping controller design of mechanical parts and electrical parts [10].

Assuming the turbine speed is given by $\omega_{w}^{*}$, the tracking error and its derivative value is

$$
\begin{aligned}
& e_{1}=\omega_{w}-\omega_{w}^{*} \\
& \dot{e}_{1}=\dot{\omega}_{w}-\dot{\omega}_{w}^{*}
\end{aligned}
$$

Define

$$
\alpha_{1}=c_{1} e_{1}
$$

where $c_{1}>0$ must be Hurwitz. Let the Lyapunov function

$$
\begin{aligned}
V_{1} & =e_{1}^{2} / 2 \\
\text { Define } & e_{2}=\dot{e}_{1}+\alpha_{1}
\end{aligned}
$$

Select the Lyapunov function as

$$
V_{2}=V_{1}+\sigma_{1}^{2} / 2
$$

where $\sigma$ is the sliding mode function, we design sliding mode function as

$$
\sigma_{1}=k_{1} e_{1}+e_{2}
$$

where $k_{1}$ must satisfy the Hurwitz condition, $k_{1}>0$.

$$
\text { Then } \begin{aligned}
\dot{V}_{2} & =\dot{V}_{1}+\sigma_{1} \dot{\sigma}_{1} \\
& =e_{1} e_{2}-c_{1} e_{1}^{2}+\sigma_{1}\left(k_{1} \dot{e}_{1}+\dot{e}_{2}\right) \\
& =e_{1} e_{2}-c_{1} e_{1}^{2}+\sigma \Theta
\end{aligned}
$$

where $A=-k k_{1} n_{g} T-k_{1} \dot{\omega}_{w}^{*}-\ddot{\omega}_{w}^{*}+\dot{\alpha}_{1} ; E=k_{1} \omega_{w}-\dot{\omega}_{w}$;

$$
\begin{aligned}
& C=k_{1} \omega_{w}^{2}+2 \dot{\omega}_{w} ; B=k_{g}\left(d T / d t-h u_{T}\right) \\
& \Theta=A-B / J_{T}+K_{o p t} C / J_{T}-K_{T} E / J_{T}-k_{g} h u_{T} / J_{T}
\end{aligned}
$$

The controller can be designed as

$$
u_{T}=J_{T}\left(A-B / J_{T}+K_{o p t} C / J_{T}-K_{T} E / J_{T}\right) / k n_{g} h
$$

Because equation (10) comprising the rotational inertia of the turbine, parameter $K_{\text {opt }}$ and damping $K_{T}$, and these quantities are as wind conditions change. Uncertainty for this parameters, using the estimated values of these parameters.

Define $\quad\left\{\begin{array}{l}F=K_{\text {opt }} / J_{T} \\ \Gamma=K_{T} / J_{T}\end{array}\right.$

Estimation error is defined as

$$
\left\{\begin{array}{l}
\tilde{J}_{T}=\hat{J}_{T}-J_{T} \\
\tilde{F}=\hat{F}-F \\
\tilde{\Gamma}=\hat{\Gamma}-\Gamma
\end{array}\right.
$$

Therefore,

$$
\left.u_{T}=\hat{J}_{T}\left(A-B / \hat{J}_{T}+\hat{F} C-\hat{\Gamma} E+L\right)\right) / k n_{g} h
$$

where $L_{1}=m\left(\sigma_{1}+\beta \operatorname{sgn}\left(\sigma_{1}\right)\right.$

The adaptive law is designed as

$$
\begin{gathered}
\dot{\hat{J}}=\sigma_{1} \gamma_{1}\left(-A-\hat{F} C+\hat{\Gamma} E-L_{1}\right) \\
\dot{\hat{F}}=-\sigma_{1} \gamma_{2} C \\
\dot{\hat{\Gamma}}=\sigma_{1} \gamma_{3} E
\end{gathered}
$$

Select the Lyapunov function as

$$
\begin{gathered}
V_{3}=V_{2}+\tilde{J}_{T}^{2} / 2 J_{T} \gamma_{1}+\tilde{F}^{2} / 2 \gamma_{2}+\tilde{\Gamma} / 2 \gamma_{3} \\
\text { Then } \quad \dot{V}_{3}=e_{1} e_{2}-c_{1} e_{1}^{2}-m \sigma_{1}^{2}-m \beta\left|\sigma_{1}\right|
\end{gathered}
$$

$$
=-e^{T} Q_{1} e-m \beta\left|\sigma_{1}\right| \leq 0
$$

where $e^{T}=\left[\begin{array}{ll}e_{1} & e_{2}\end{array}\right], Q_{1}=\left[\begin{array}{cc}c_{1}+m k_{1}^{2} & m k_{1}-1 / 2 \\ m k_{1}-1 / 2 & m\end{array}\right]$

By taking $m, c_{1}$ and $k_{1}$ value, enables the $|Q|$ is greater than zero, thereby ensuring $Q$ is a positive definite matrix.

Assumed turbines ideal position signal of flux is constant, The tracking error's derivative value is

$$
\begin{array}{cc} 
& \dot{z}_{1}=\dot{\psi}-\dot{\psi}^{*}=f x-2 e \psi \\
\text { Define } & \alpha_{2}=c_{2} z_{1}, c_{2}>0
\end{array}
$$

Select the Lyapunov function as

$$
\begin{gathered}
S_{1}=z_{1}^{2} / 2 \\
\text { Define } \quad z_{2}=\dot{z}_{1}+\alpha_{2}
\end{gathered}
$$

Select the Lyapunov function as

$$
S_{2}=S_{1}+\sigma_{2}^{2} / 2
$$

where $\sigma_{2}$ is the sliding mode function, we design sliding mode function as

$$
\sigma_{2}=k_{2} z_{1}+z_{2}
$$


In order to avoid using the upper bound of disturbance $\mathrm{D}$, we use adaptive algorithm to estimate the disturbance D.

Select the Lyapunov function as

$$
S_{3}=S_{2}+\tilde{D}^{2} / 2 \gamma_{4}
$$

where $\gamma$ is positive constant, $\hat{D}$ is the estimate of $D$, estimate error is $\tilde{D}=D-\hat{D}$.

The adaptive law is designed as

$$
\dot{\hat{D}}=\sigma_{2} f \gamma_{4}
$$

The controller can be designed as

$$
\left.u_{\psi}=\left(-M-f N-f \hat{D}+\dot{\alpha}_{2}-t L_{2}\right)\right) / f h
$$

where $N=\dot{x}-D-h u_{\psi} \quad, \quad L_{2}=\sigma_{2}+\varepsilon \operatorname{sgn}\left(\sigma_{2}\right)$,

$$
M=\left(k_{1}-2 e\right) \dot{\psi} \text {. }
$$

$$
\begin{aligned}
\dot{S}_{3} & =\dot{S}_{2}-\tilde{D} \dot{\hat{D}} / \gamma_{4} \\
& =-Z^{T} Q_{2} Z-t \varepsilon\left|\sigma_{2}\right| \leq 0
\end{aligned}
$$

$$
\text { where } z^{T}=\left[\begin{array}{ll}
z_{1} & z_{2}
\end{array}\right], Q_{2}=\left[\begin{array}{cc}
c_{2}+t k_{2}^{2} & t k_{2}-1 / 2 \\
t k_{2}-1 / 2 & t
\end{array}\right]
$$

In order to restrain chattering phenomenon, the use of a relay function for obtaining the alternate control input induces unacceptable generator torque or current variations. Use of a continuous hyperbolic tangent function is a well-know method for alleviating the supplementary fatigue due to current oscillations and electromagnetic torque without greatly affecting the control law robustness.

\section{SimUlATION RESUlTS}

To evaluate the performance of the proposed adaptive sliding mode backstepping control algorithm, the proposed sliding mode torque regulation strategy has been tested for validation using the NREL FAST and TurbSim code [11].

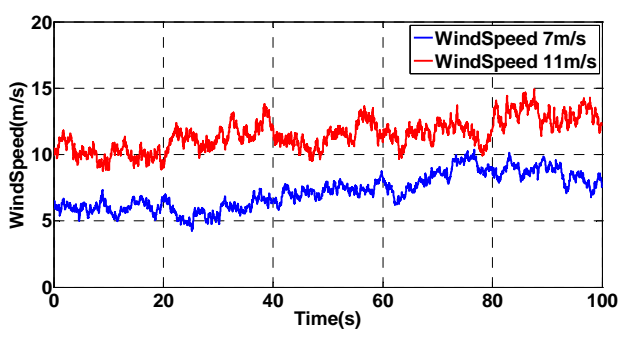

Figure 2. Wind speed profile

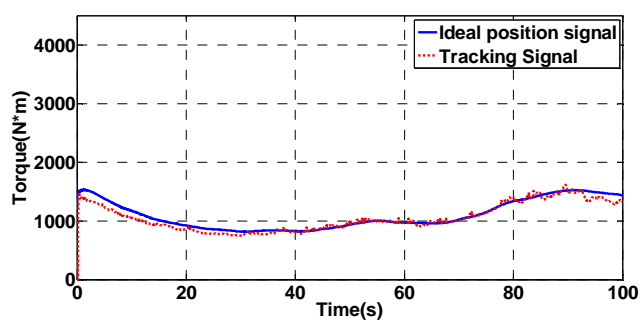

Figure. 3. Torque: reference (blue) and real (red)
Numerical validations, using FAST and TurbSim with MATLAB has been carried out on the NERL CART-3 WT. Validation tests were performed using turbulent wind data with 7 and $11 \mathrm{~m} / \mathrm{s}$ wind speeds respectively see the Figure 2 . As clearly shown in Figs. 3, 4, very good tracking performances are achieved in terms of DFIG torque with respect to wind fluctuations.

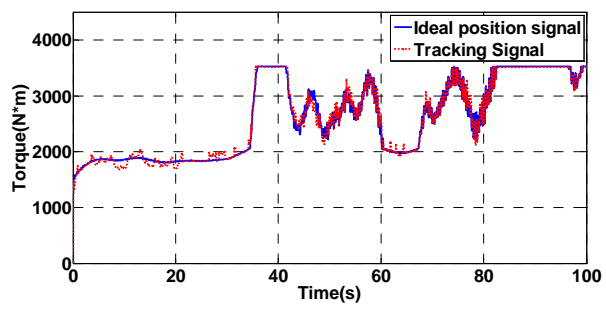

Figure 4. Torque: reference (blue) and real (red)

\section{CONCLUSION}

A model-independent control scheme based on adaptive backstepping slide control method is developed for torque tracking of DFIG system. Based on Lyapunov stability theory, we establish torque and flux tracking of proposed adaptive slide control scheme. The proposed sliding mode control strategy present attractive features such as robustness to parametric uncertainties of the turbine and the generator as well as to electric grid disturbances. The obtained results clearly show the adaptive slide control approach effectiveness in terms of power extraction maximization compared to more traditional technique.

\section{REFERENCES}

[1] T. Ackermann, Wind Power in Power Systems, West Sussex: John Wiley \& Sons, 2005, p. 8.

[2] “Renewables 2012 Global Status Report” [Online] Global wind energy council, Available: http//www.gwec.net

[3] S. Bacha, J, Guiraud. "Energy-Reliability Optimization of Wind Energy Conversion System by Sliding Mode Control” , IEEE Transl. J. Energy convers., vol. 23, no. 3, pp. 975-985, Sep 2008

[4] B. Beltran, E. Hachemi Benbouzid, M. Ahmed-Ali, "Second-Order Sliding Mode Control of a Doubly Fed Induction Generator Driven Wind Turbine”, IEEE Transl. J. Energy convers., vol. 27, no. 2, pp. 261-269, June 2012

[5] A. Khazaee, H. A. Zarchih, M. Ebrahimi, "Robust Maximum Power Point Tracking Control of Permanent Magnet Synchronous Generator for Grid Connected Wind Turbine”, IEEE Transl. J. Renewable Energy and Distibuted Generation, pp. 75-79, March 2012

[6] D. Rush, D. G. Wilson, Nonlinear Power Flow Control Design, London, U.K.:Springer, pp. 274-276, Aug 2011

[7] S. E. Ben, M. E. Hachemi, "High-order Sliding Mode Control of a Marine Current Turbine Driven Doubly-Fed Induction Generator", IEEE Transl. J. Ocean Engineering., vol. 35, no. 2, pp. 402-411, April 2010

[8] W. Jiuhe, Nonlinear Control of AC motor, Beijing: Electronics industry Press, pp. 10-24, Aug 2009

[9] P. J. Darrow, Wind Turbine Control Design to Reduce Captial Costs Natl. Renew. Energy Lab, Golden, CO, NREL, Aug 2009

[10] K. J. Åstrom, B. Wittenmark, Adaptive Control, 2nd ed., Beijing, Science Press. March 2003

[11] J. M. Jonkman, M. L. Buhl, “FAST user's guide,” Natl. Renew. Energy Lab, Golden, CO, NREL/EL-500-38230, Aug 2005 